\section{Atenção ao pré-natal e parto em mulheres usuárias do sistema público de saúde residentes na Amazônia Legal e no Nordeste, Brasil 2010}

\section{Prenatal and childbirth care for women using the public health system resident in Amazonia Legal and the Northeast Region of Brazil 2010}

\footnotetext{
Abstract

Objectives:to describe the adequacy of healthcare among women undergoing prenatal and/or childbirth care in the Brazilian National Health System, SUS, in municipalities that have been earmarked for reduction of infant mortality in Amazonia Legal and the Northeast Region.

Methods: secondary data from a populationbased survey involving mothers and children aged under one year of age attended by the 2010 vaccination campaign were analyzed. The sample under study comprised 13.205 women who had received prenatal care and 13,044 whose deliveries had been accompanied, in 252 earmarked municipalities. The adequacy of prenatal and childbirth care was classified according to process indicators proposed by the National Program for the Humanization of Prenatal Care and Childbirth.

Results: of the women studied, $75.4 \%$ had attended six or more prenatal consults, but only $3.4 \%$ had access to prenatal care classified as adequate. Access to ultrasound was reported by $96.1 \%$ of the women, an HIV exam by $91.8 \%$ and a syphilis test by $68.7 \%$. Only $44.2 \%$ of the women were told which maternity hospital they should give birth in and only $8.6 \%$ were in fact admitted to the recommended facility. Childbirth care was considered adequate for only $1 \%$ of those interviewed. The results varied from one State to another and according to the socioeconomic status of the women.

Conclusions: shortcomings were identified in prenatal and childbirth care, which is inadequate and socially unjust in these regions, thereby contributing to poor indicators for maternal and child health in Legal Amazonia and the Northeast Region of Brazil. Key words Prenatal care, Maternal welfare, Health services accessibility, Quality of health Care, Maternal mortality
}

Maria do Carmo Leal 1

Mariza Miranda Theme-Filha 2

Erly Catarina de Moura 3

José Guilherme Cecatti 4

Leonor Maria Pacheco Santos 5

1,2 Escola Nacional de Saúde Pública. Fundação Oswaldo Cruz. Rua Leopoldo Bulhões, 1480. Manguinhos. Rio de Janeiro, RJ, Brasil. CEP: 21.041-210. E-mail: leopac@unb.br

3 Fundação Oswaldo Cruz. Rio de Janeiro, RJ, Brasil.

4 Departamento de Tocoginecologia. Faculdade de Ciências Médicas. Universidade Estadual de Campinas. Campinas, SP, Brasil.

5 Departamento de Saúde Coletiva. Universidade de Brasília. Brasília, DF, Brasil.

Resumo

Objetivos: descrever a adequação da atenção à saúde entre as mulheres que fizeram o pré-natal elou parto no Sistema Único de Saúde (SUS), nos municípios prioritários para a redução da mortalidade infantil na Amazônia Legal e no Nordeste.

Métodos: análise de dados secundários de inquérito de base populacional com mães e crianças menores de um ano de idade que compareceram à Campanha de vacinação em 2010. A amostra estudada foi de 13.205 mulheres com acompanhamento de pré-natal e de 13.044 mulheres com acompanhamento de parto, em 252 municípios prioritários. A adequação do pré-natal e parto foi classificada em conformidade com indicadores de processo propostos pelo Programa Nacional de Humanização do Prénatal e Nascimento.

Resultados: entre as mulheres investigadas 75,4\% realizaram seis ou mais consultas de pré-natal, mas somente 3,4\% tiveram acesso a um pré-natal classificado como adequado. $O$ acesso à ultrassonografia foi relatado por $96,1 \%$ das mulheres, ao exame de HIV por 91,8\% e ao teste de sifilis por 68,7\%. Apenas 44,2\% das mulheres recebeu indicação da maternidade na qual deveria fazer o parto e a internação no local indicado ocorreu em 8,6\% dos casos. A atenção ao parto foi considerada adequada para apenas $1 \%$ das entrevistadas. Os resultados variaram entre os estados e níveis socioeconômicos das mulheres.

Conclusões: foram identificadas falhas na atenção ao pré-natal e parto, que é inadequada e socialmente iníqua nestas regiões, contribuindo para os precários indicadores de saúde materno infantil na Amazônia Legal e no Nordeste do Brasil.

Palavras-chave Assistência pré-natal, Saúde materna, Acesso aos serviços de saúde, Qualidade da assistência à saúde, Mortalidade materna 


\section{Introdução}

A redução da mortalidade materna e infantil requer a oferta de serviços de qualidade que garantam acesso facilitado, cumprimento dos protocolos estabelecidos baseados nas melhores evidências científicas e elevada cobertura populacional, além de proporcionar intervenções oportunas que visem garantir a saúde materna, em particular das gestantes. ${ }^{1}$

A assistência pré-natal é um fator de proteção para a saúde da mãe por incluir procedimentos rotineiros preventivos, curativos e de promoção da saúde. Quando bem conduzida pode contornar problemas obstétricos, prevenir danos e assegurar partos e nascimentos saudáveis. Revisões sistemáticas demonstram a efetividade de diversas práticas realizadas rotineiramente na assistência pré-natal para prevenção da morbimortalidade materna e perinatal.2,3

Em 2000, o Ministério da Saúde lançou o Programa de Humanização do Pré-Natal e Nascimento (PHPN), com o objetivo de desenvolver ações de promoção, prevenção e assistência à saúde de gestantes e recém-nascidos, promovendo a ampliação do acesso, o incremento da qualidade e da capacidade instalada da assistência obstétrica e neonatal, bem como sua organização e regulação no âmbito do Sistema Único de Saúde (SUS). O principal objetivo do PHPN é fornecer uma assistência de qualidade e humanizada à gestante e ao recémnascido, estabelecendo bases referenciais para a promoção de uma assistência pré-natal de qualidade em todo Brasil.4

A avaliação da qualidade da atenção pré-natal é um passo importante para medir sua efetividade e o impacto nos indicadores de resultados maternos $\mathrm{e}$ neonatais. A construção dos índices nacionais deve ser baseada nos critérios estabelecidos pelo PHPN, que define um conjunto de exames laboratoriais, administração de vacinas, realização de atividades educativas, classificação de risco e encaminhamento dos casos de riscos.

Apesar da cobertura quase universal de consultas pré-natais no Brasil, 5 avaliações sobre a qualidade da assistência têm revelado baixa qualidade.6,7 Estudo sobre os óbitos por causas evitáveis em menores de um ano no Brasil no período 1997-2006 encontrou redução de todas as mortes evitáveis, exceto aquelas relacionadas à uma adequada atenção pré-natal, sugerindo baixa qualidade dessa assistência além de grandes iniquidades segundo características socioeconômicas das mulheres. ${ }^{8}$

A cobertura da atenção ao parto hospitalar também alcança 98\% das mulheres, apesar dos elevados índices da razão da mortalidade materna e perinatal, sugerindo problemas na qualidade da atenção ofertada. ${ }^{9} \mathrm{Na}$ última década a atenção ao parto tem sido alvo de políticas públicas que incluem desde a mudança do modelo de atenção obstétrica, como a diminuição das intervenções sobre o parto vaginal quanto à redução das cesarianas desnecessárias.

Um dos aspectos priorizados no contexto dessas políticas públicas foi a criação, em 2005, da Lei do direito ao acompanhante, ${ }^{10}$ que se aplica a todas as mulheres no serviço público ou privado. Uma revisão sistemática recente sobre o efeito da presença contínua do acompanhante mostrou que a intervenção se relaciona com a prevenção de cesarianas, menor uso de analgesia epidural, satisfação materna e valores adequados do escore de Apgar no quinto minuto para o recém-nascido. 11

Outro aspecto a ser destacado é a falta de continuidade entre a atenção pré-natal e ao parto no país, deixando as gestantes sem referenciamento para uma maternidade de fácil acesso e adequada para as suas necessidades clínicas. A Lei $\mathrm{n}^{\circ} 11.634 /$ 2007, passa a garantir às gestantes atendidas no Sistema Único de Saúde o direito de vincular-se à maternidade onde receberá assistência ao parto. 12 Ainda em relação ao parto, evidências sobre a atenção ao parto de risco obstétrico habitual são unanimes em propor um reforço à autonomia das mulheres na condução do seu próprio parto e um manejo que inclui a liberdade de movimentos e promoção do bem estar, denominado de boas práticas obstétricas, ficando os serviços de saúde com o papel de suporte para o desenvolvimento do trabalho de parto e parto. 13,14

O Ministério da Saúde, por meio da Portaria ${ }^{\circ}$ 1.459/2011 instituiu a Rede Cegonha como estratégia para organizar uma rede de cuidados que assegurasse às mulheres o direito ao planejamento reprodutivo e à atenção humanizada à gravidez, ao parto, ao puerpério e o direito ao nascimento e ao desenvolvimento saudável das crianças. 15

O contexto de baixa autonomia das mulheres, de tantas intervenções obstétricas, de peregrinação na busca do atendimento ao parto e de inadequados indicadores perinatais se constituem num cenário de violência obstétrica que redunda em desatenção e discriminação social.5,16

Este artigo tem por objetivo descrever a adequação da atenção ao pré-natal e ao parto recebida pelas usuárias do Sistema Único de Saúde em 252 municípios prioritários para a redução da mortalidade infantil na Amazônia Legal e no 
Nordeste do Brasil, a partir de indicadores estratégicos para a redução da mortalidade perinatal. O estudo, realizado em 2010, oportuniza a construção de uma linha de base para Rede Cegonha em municípios prioritários para a redução da mortalidade infantil.

\section{Métodos}

Este estudo analisou dados secundários da pesquisa "Chamada Neonatal" inquérito de base populacional que avaliou 16.863 pares de mães e crianças menores de um ano de idade que compareceram à Campanha Nacional de Vacinação no dia 12 de junho de 2010 em 252 municípios prioritários. O método, incluindo o treinamento, controle de qualidade, o cálculo dos fatores de expansão da amostra e as perdas amostrais, é descrito detalhadamente na publicação oficial: "Avaliação da atenção ao prénatal, ao parto e aos menores de um ano na Amazônia Legal e no Nordeste, Brasil, 2010".17 A coleta de dados ocorreu em 252 dos 256 municípios prioritários para a Redução da Mortalidade Infantil no Nordeste e na Amazônia Legal (98\%).Um município da Amazônia não participou do inquérito pelo fato de haver predominantemente população indígena e três municípios do Nordeste pela falta de recursos humanos no local. Foi possível produzir estimativas para os dezessete estados, suas capitais e ainda estimativas para o conjunto de municípios do interior de cada Estado.17

Para analisar a atenção ao pré-natal considerouse como usuária do SUS a mulher que respondeu ter realizado o pré-natal em serviços exclusivamente públicos, em um total de 13.205 (78,3\% da amostra total, sendo 5081 na Amazônia Legal e 8124 no Nordeste) e, para a análise da atenção ao parto, a mulher que respondeu ter utilizado serviço público para a realização do parto, em um total de 13.044 (77,4\% da amostra total, sendo 4922 na Amazônia Legal e 8122 no Nordeste). Dos Estados avaliados, o menor percentual de mulheres que foram atendidas nos serviços do SUS foi observado em Mato Grosso (70,6\% no pré-natal e $63,6 \%$ no parto) e os maiores no Acre (91,0\% no pré-natal) e em Roraima (91,6\% no parto)

As análises foram realizadas com a amostra expandida; calculou-se a proporção e o Intervalo de Confiança de $95 \%$ das variáveis de interesse. O indicador de conformidade da assistência prestada no pré-natal foi classificado em três categorias com base nos indicadores de processo propostos pelo Programa Nacional de Humanização do Pré-natal e Nascimento (PHPN).15 Adequada: início do pré- natal no primeiro trimestre gestacional, número de consultas igual ou maior que seis; aferição da pressão arterial e do peso em todas as consultas e exame de mamas em pelo menos uma consulta; realização de hemograma, glicemia e exame de urina; realização de teste para sífilis (VDRL-venereal disease research laboratory) e Aids (HIV-human immunodeficiency virus) com entrega do resultado em até 15 dias; recebimento de orientação sobre aleitamento materno e sobre o local do parto. Parcialmente adequada se houve pelo menos uma das seguintes ocorrências: início do pré-natal no segundo trimestre gestacional, número de consultas entre três e cinco; aferição da pressão arterial, do peso e exame das mamas em pelo menos uma consulta; ter realizado pelo menos três dos cinco exames laboratoriais acima listados, podendo não ter recebido orientação sobre aleitamento ou local do parto. Inadequada se houve pelo menos uma das seguintes ocorrências: início do pré-natal no terceiro trimestre gestacional, número de consultas menor que três e ter realizado menos de três dos cinco exames recomendados.

Para analisar a qualidade da assistência recebida no parto, o indicador foi construído a partir de variáveis que retratam a peregrinação e a humanização no atendimento, descritos a seguir. Adequada: realização do parto na maternidade indicada durante o pré-natal, no primeiro serviço procurado, espera para internação menos de uma hora e acompanhante em todos os momentos do parto. Parcialmente adequada: realização do parto no local indicado, no primeiro serviço procurado, espera para internação menos de uma hora e acompanhante em um ou dois momentos. Inadequada: não ser atendida no primeiro serviço que procurou, ou esperar mais de uma hora para internação, ou parir em outro município, ou não ter tido acompanhante em nenhum momento do parto.

Os indicadores de adequação da assistência no pré-natal e no parto foram analisados segundo variáveis sociodemográficas (idade materna, escolaridade materna, raça/cor da pele materna, chefe da família, localidade da residência) e tempo de duração de recebimento do benefício pelo Bolsa Família. A associação entre qualidade de assistência e as variáveis sociodemográficas foi realizada por meio do teste do qui-quadrado (para tendência - se apropriado), considerando-se nível de significância de $5 \%$.

\section{Resultados}

A Tabela 1 mostra que, das mulheres que realizaram 
o pré-natal em serviços públicos, a maioria iniciou no primeiro trimestre de gestação $(75,8 \%)$ e realizou seis ou mais consultas $(75,4 \%)$, não havendo diferença entre as Regiões estudadas. Quanto aos indicadores de cuidado, $99,2 \%$ das mulheres relataram aferição da pressão arterial e 99,0\% destas indicaram ter sido realizada a aferição do peso em todas as consultas. Porém apenas 50,2\% referiram ter realizado exame das mamas, com situação mais favorável na Região Nordeste. Os exames laboratoriais mais frequentemente apontado pelas entrevistadas foram urina $(96,8 \%)$ e hemograma $(93,0 \%)$, com a Região da Amazônia Legal apresentando as maiores proporções, e a glicemia que cobriu $83,9 \%$. O teste para HIV foi relatado por $91,8 \%$ das mulheres, embora apenas $40,9 \%$ tenham recebido os resultados no prazo de 15 dias. No entanto $68,7 \%$ responderam ter realizado teste de sífilis e 47,0\% receberam o resultado em quinze dias. Em ambos os casos a pior situação para entrega de resultados registrou-se entre as mulheres da Região Nordeste. $\mathrm{O}$ acesso à ultrassonografia obstétrica ocorreu em $96,1 \%$ dos casos, com uma média de 3,0 exames por gestante. O recebimento de orientação sobre aleitamento materno foi relatado por $78,6 \%$ das mulheres na Região Nordeste. No entanto, a indicação do local do parto ocorreu em $44,2 \%$ dos casos, sendo maior também na Região Nordeste.

Nesta mesma Tabela 1 verifica-se que, das mulheres que realizaram o parto em serviços públicos, apenas $8,6 \%$ foram atendidas no serviço indicado durante o pré-natal, sem diferença entre as regiões. A maioria $(80,5 \%)$ das gestantes realizou o parto na primeira maternidade procurada, sendo observado maior percentual na Amazônia Legal $(83,8 \%)$. Somente $36,6 \%$ esperaram menos de uma hora para serem hospitalizadas. A utilização de serviços de outro município foi o triplo no Nordeste $(17,9 \%)$ em relação à Amazônia Legal (5,7\%). O parto cesário ocorreu em $40,9 \%$ dos casos, sem diferenças entre as Regiões. A ausência de acompanhante durante o pré-parto, parto e pós-parto foi de aproximadamente de $20,0 \%$ em ambas as regiões, sendo o mesmo percentual de mulheres que estiveram acompanhadas em todos os momentos da hospitalização para o parto (maior na Amazônia Legal).

A Tabela 2 descreve a adequação do pré-natal para cada Estado. No total apenas 3,4\% das mulheres tiveram acesso a um pré-natal classificado como adequado, $88,6 \%$ como parcialmente adequado e $8,0 \%$ como pré-natal inadequado. A menor frequência de pré-natal adequado foi observada no Estado do Acre (1,5\%) e a maior no Rio Grande do
Norte $(6,3 \%)$. Destacam-se os Estados de Alagoas e Maranhão com mais de $10 \%$ de pré-natal inadequado.

O pré-natal adequado foi mais comum entre as mulheres com 30 e mais anos de idade, com escolaridade acima de dez anos de estudo, entre as mulheres residentes nas capitais e sem benefício do Programa Bolsa Família (Tabela 3). Já a inadequação foi maior entre as mulheres com menos de 20 anos de idade, com baixa escolaridade, residentes no interior e entre as que recebiam o benefício do Programa Bolsa Família há menos de um ano. Não houve diferença quanto à raça/cor da pele materna e ser chefe da família.

A Tabela 4 mostra que a classificação da atenção ao parto foi considerada inadequada em $99,0 \%$ dos casos, sem diferença entre os Estados avaliados. A proporção de partos classificados como adequados foi irrisória $(0,2 \%)$, chegando a não constar nenhuma observação no Mato Grosso, Tocantins e Bahia, assim como os parcialmente adequados $(0,8 \%)$, com nenhuma observação em Roraima.

As variáveis sociodemográficas idade, escolaridade, raça/cor da pele, ser chefe de família, residir na capital ou interior e ter o benefício do Programa Bolsa Família foram analisadas em relação à adequação da atenção ao parto (Tabela 5). Verificase que a idade e a escolaridade da mãe, o local de residência e duração do benefício Bolsa Família se associaram com adequação da atenção ao parto. As mulheres com mais idade, as mulheres mais instruídas e as mulheres que não recebem Bolsa Família têm os melhores resultados, mas residir nas capitais dos Estados mostrou-se desfavorável.

\section{Discussão}

A técnica adotada na pesquisa original da Chamada Neonatal foi a coleta de dados descentralizada em 252 municípios em um só dia. Apresenta como principais vantagens o baixo custo, a rapidez na coleta e a mobilização dos gestores locais para a pesquisa em todos os municípios prioritários. Por outro lado, apresenta as limitações inerentes aos estudos de prevalência pontuais, somadas aos desafios logísticos de uma operação deste porte, para garantir a coleta de dados de boa qualidade. Os resultados obtidos propiciam tanto avanços no conhecimento, como subsidiam o planejamento e gestão dos serviços de saúde.

Os investimentos na melhoria da atenção à saúde das mulheres, adolescentes e crianças nas últimas décadas resultaram em avanços importantes, expressos nos diversos indicadores de saúde destas 


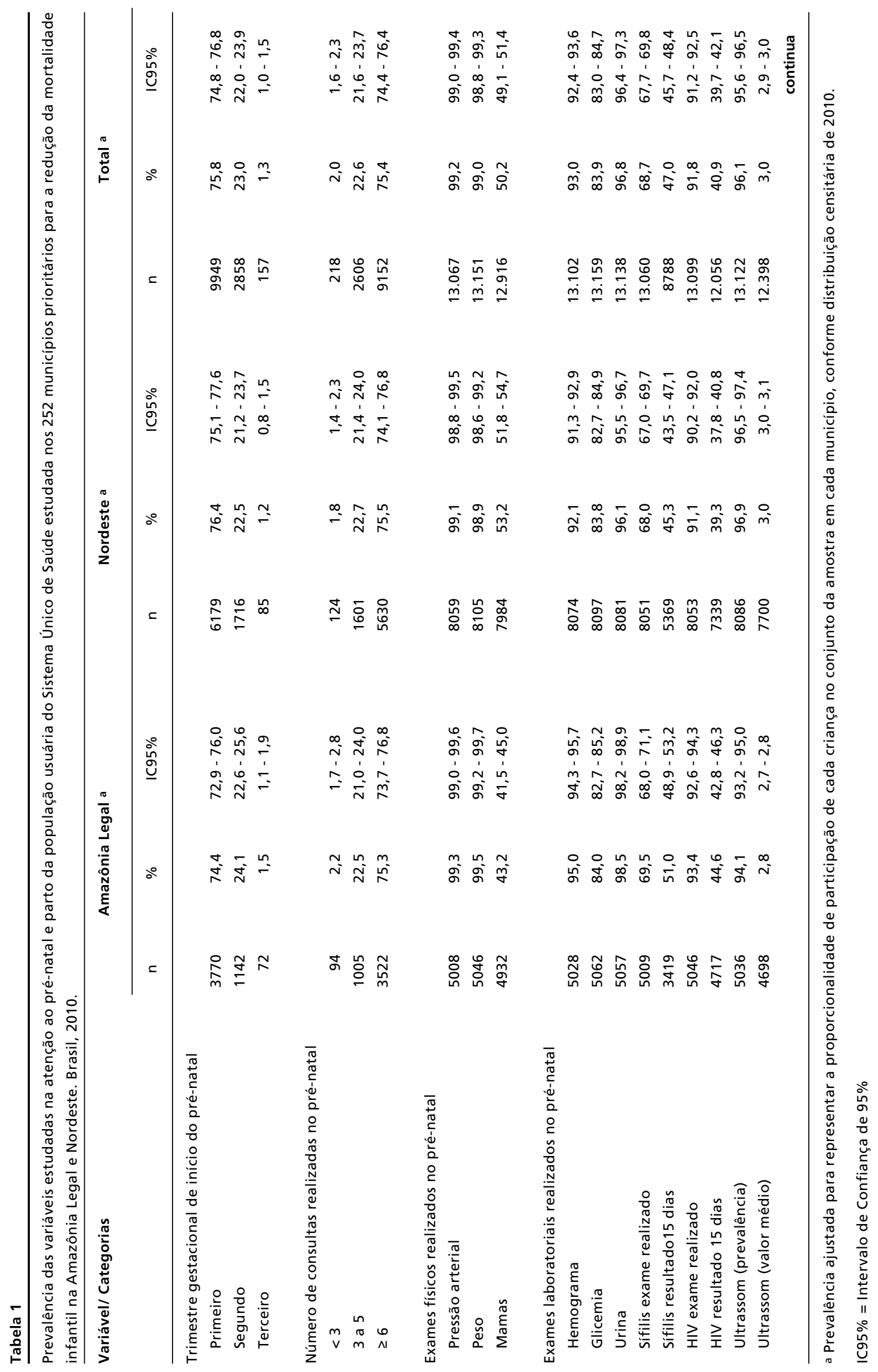




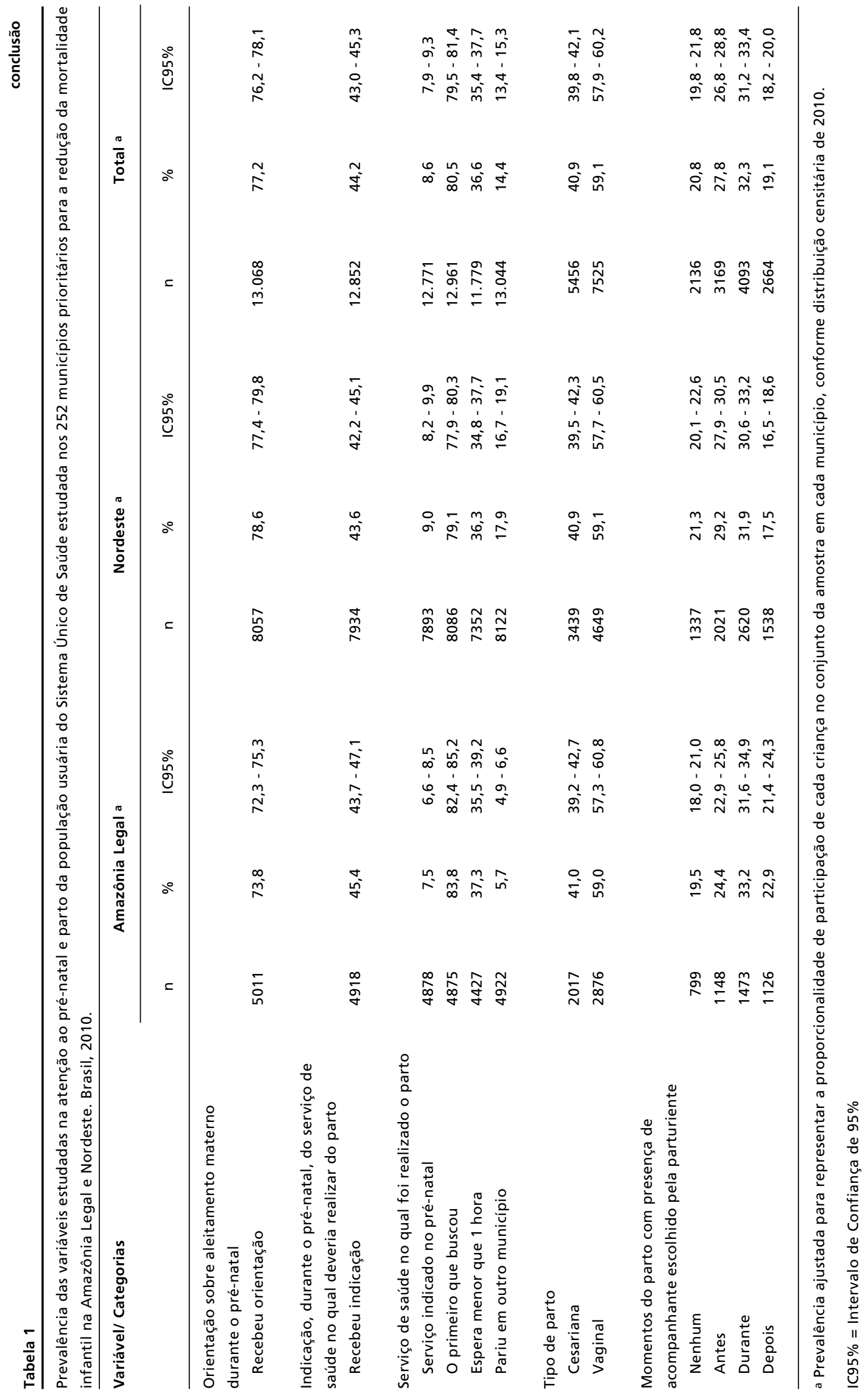




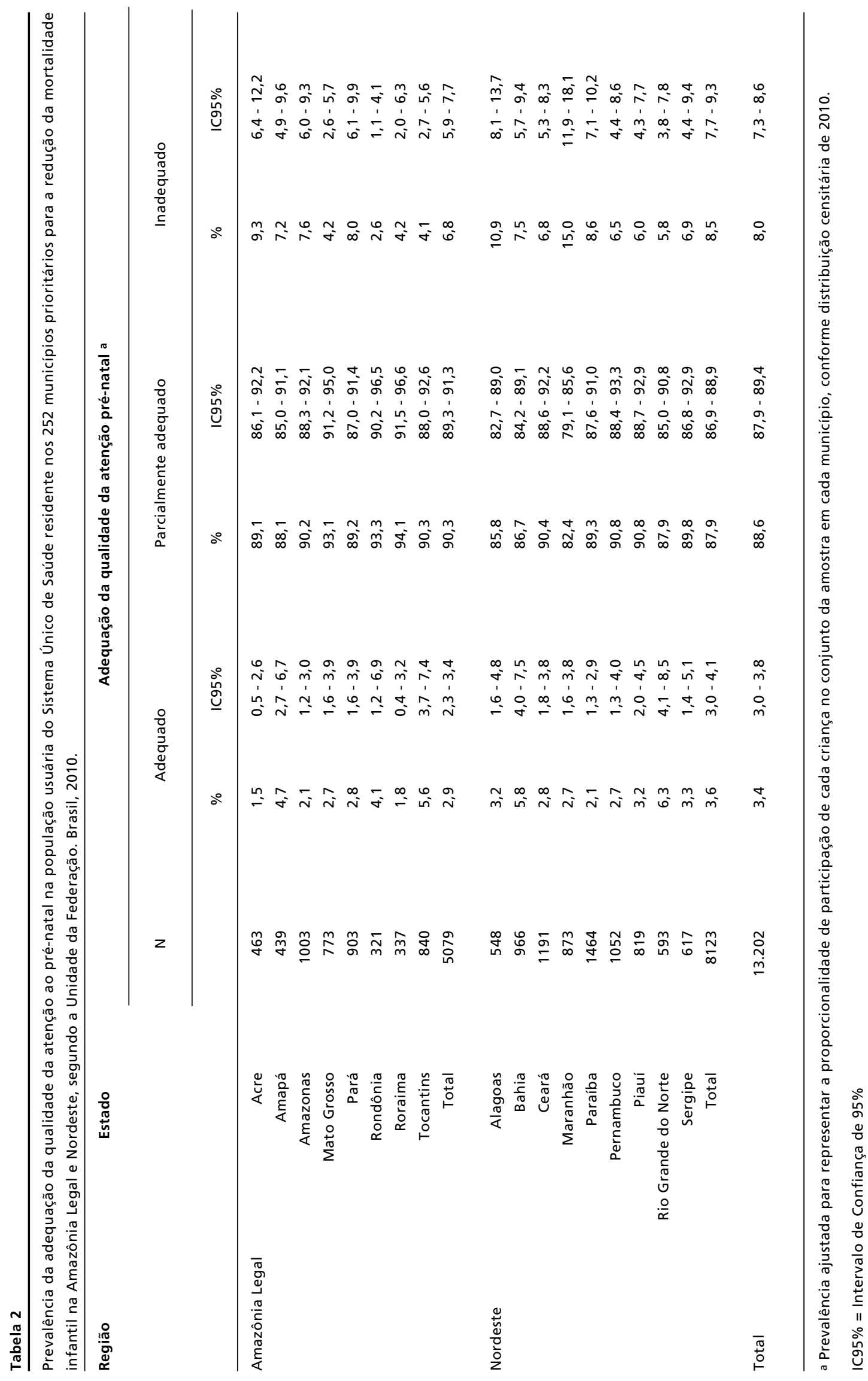




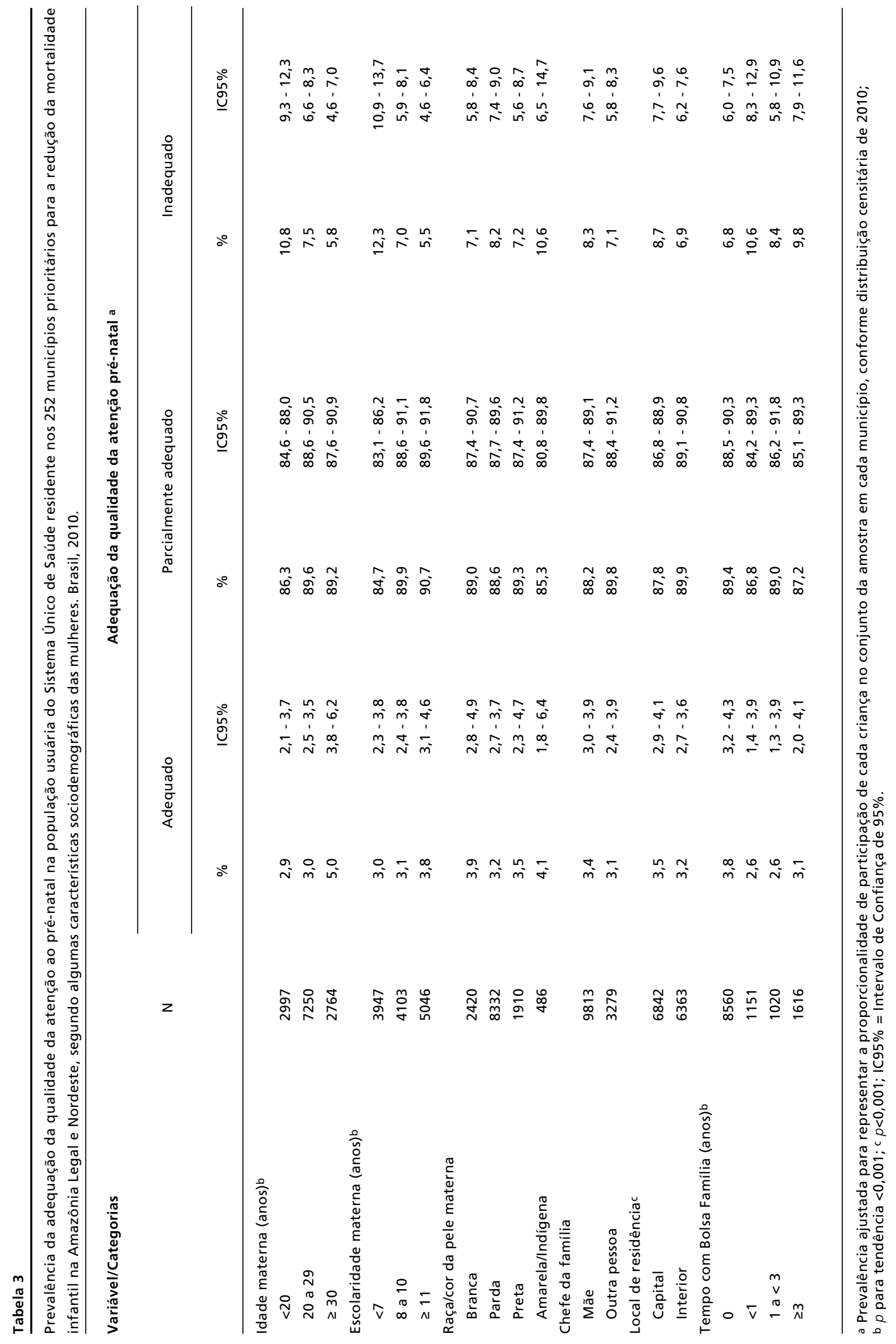




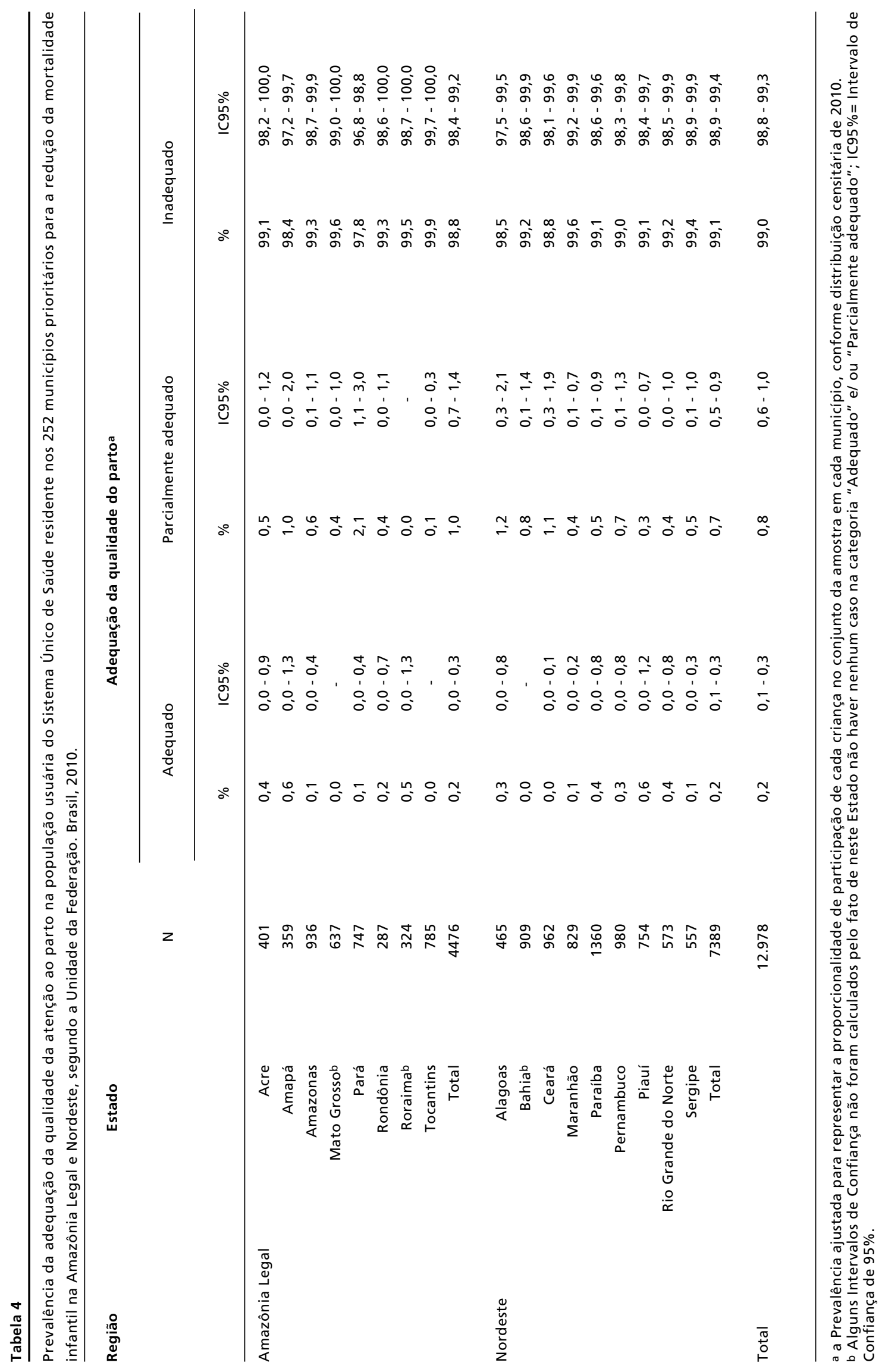




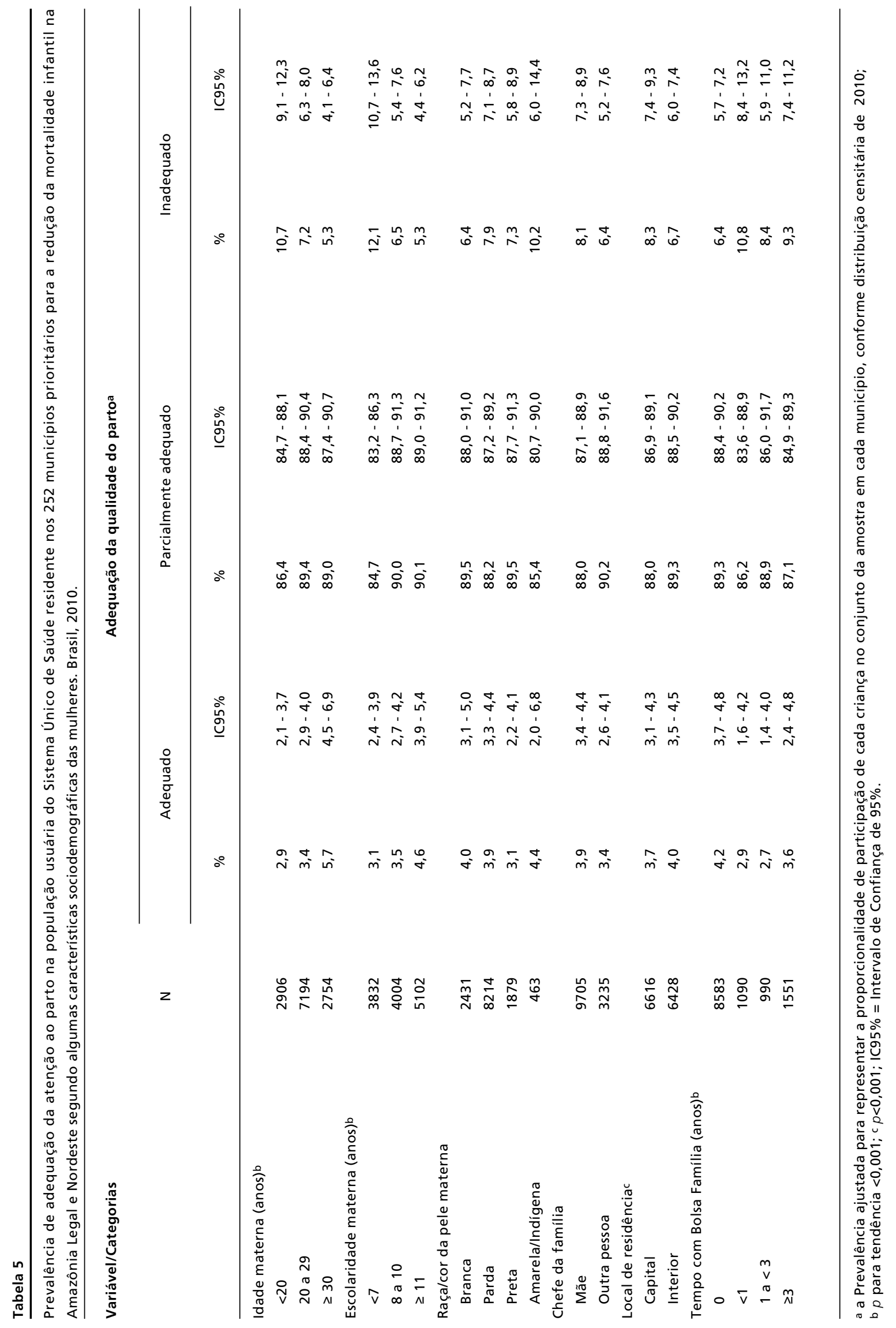


populações. ${ }^{19}$ Entretanto, indicadores relacionados especificamente à saúde materna e perinatal ainda permanecem aquém daqueles encontrados em alguns países em desenvolvimento e muito distante dos indicadores dos países desenvolvidos. Mantido o ritmo atual de decréscimo da Razão de Mortalidade Materna (RMM), o Brasil não conseguirá atingir a Meta de Desenvolvimento do Milênio (ODM) número 5 , de redução de três quartos da mortalidade materna entre 1990 e 2015.20 Em relação aos ODM relativos à saúde das crianças, ainda que se alcance a meta para o país, grandes disparidades regionais persistem, em especial na Região Nordeste e na Amazônia Legal, permanecendo como um desafio para a gestão pública.

Entre os fatores relacionados para o Brasil não alcançar as metas pactuadas destaca-se a persistente baixa qualidade da assistência pré-natal e ao parto. Apesar das evidências da importância da atenção pré-natal na prevenção e detecção precoce de agravos, e consequente redução da morbi-mortalidade materna e infantil,21,22 a qualidade da atenção permanece questionável. Os primeiros estudos avaliativos nacionais do Programa de Humanização do Pré-Natal e Nascimento (PHPN) revelaram que apenas $22,0 \%$ das mulheres realizaram seis ou mais consultas de pré-natal e que somente 5,6\% realizaram o conjunto de atividades assistenciais estabelecidas, mostrando um perfil de assistência desarticulada e parcial.23 Estudos realizados em Juiz de Fora, MG (2002/2004)24 e no Rio de Janeiro $(2007 / 2008)^{25}$ evidenciaram a persistência de falhas no atendimento, a despeito da elevada cobertura de consultas pré-natais com índices globais de qualidade não atingindo $40,0 \%$ das gestantes atendidas no SUS. Os dados aqui apresentados foram coletados em 2010, porém em municípios das Regiões mais pobres do Brasil. Mostraram que os avanços alcançados ainda se alternavam com falhas na atenção, resultando em valores de inadequação bem superiores aos encontrados nos estudos acima, realizados na Região Sudeste. No entanto os resultados são compatíveis com os precários indicadores de saúde materno infantil na Amazônia Legal e no Nordeste do Brasil.

Cabe salientar também o rigor imposto pelo conjunto de indicadores utilizados, em especial na assistência ao parto, cuja inadequação atingiu expressiva maioria destes, dificultando discriminar diferenças. Todavia, não há argumento que justifique a exclusão de qualquer um deles - não ser atendida no primeiro serviço procurado, esperar mais de uma hora para internação, parir em outro município, não ter acompanhante em nenhum momento do parto - todos indispensáveis na mensuração da qualidade de cuidado ao parto.

Este estudo mostrou que as mulheres usuárias de serviços públicos residentes nos 252 municípios prioritários para a redução da mortalidade infantil na Amazônia Legal e Nordeste tiveram níveis muito baixos de adequação da atenção ao pré-natal e ao parto. Quanto à adequação do pré-natal, de acordo com os critérios definidos para esse estudo, chama atenção a baixa proporção de pré-natal classificado como adequado, e o predomínio de classificação como parcialmente adequado. A adequação do prénatal relacionou-se com a condição social e demográfica da gestante, sendo pior para as de baixa condição social.

As variáveis que mais se relacionaram com falhas na atenção foram a não realização de exame das mamas em nenhuma consulta, não entrega dos resultados de exames de sífilis e Aids no prazo de 15 dias e ausência de referência do local do parto. Particularmente em relação ao diagnóstico da sífilis, além do atraso na entrega oportuna do resultado do exame, chama atenção a baixa proporção de realização de testagem, devido ao seu potencial impacto na prevenção da sífilis congênita, cuja prevalência tem mostrado tendência de aumento nos últimos anos. 26 Os valores extremamente baixos mostrados nesse estudo merecem uma atenção específica e cuidadosa dos gestores destas localidades. Além disso, durante o pré-natal, todas as mulheres devem ser novamente testadas para sífilis e deveriam ser informadas sobre esse e os demais procedimentos. Por outro lado o acesso ao exame de ultrassonografia obstétrica nesta pesquisa foi relatado por expressiva maioria das entrevistadas sendo, em média, realizados três exames em cada gestante, em franco contraste com a ausência de outros exames essenciais como relatado por Victora et al. 20

Com relação a outros critérios de conformidade da assistência do pré-natal, como a realização de pelo menos seis consultas e o início do acompanhamento no primeiro trimestre, ambos constituindo indicadores de processo para avaliação do pré-natal do PHPN, as proporções ponderadas foram elevadas. Estes resultados contrastam com os achados da Pesquisa Nascer no Brasil, com representatividade nacional, para as cinco regiões e para o setor público e privado, realizado entre 2011 e 2012, que mostrou que $73,1 \%$ das gestantes realizaram seis ou mais consultas pré-natais, porém com variações importantes e significativas entre as regiões, com os piores indicadores nas Regiões Norte e Nordeste $(57,3 \%$ e $65,6 \%$, respectivamente) possivelmente como reflexo de diferenças entre os métodos utilizados 
nestes estudos. Valores semelhantes foram encontrados em relação ao início precoce do pré-natal, com média nacional de $75,8 \%$, porém com valores inferiores a esta média nas Regiões Norte e Nordeste (64,4\% e $73,7 \%$, respectivamente). 5

Estudos brasileiros sobre saúde perinatal mostram que o acesso à atenção pré-natal, avaliado pelo número de consultas realizadas e o mês do início do atendimento, protege contra a prematuridade, o baixo peso ao nascer e o óbito perinatal. Estes resultados permanecem significativos mesmo depois de controlada a condição social.7,27

Embora a maioria das mulheres relate que durante o pré-natal sua pressão arterial e peso foram aferidos, apenas cerca de metade relatou que suas mamas foram examinadas, um procedimento de rotina que pode aferir igualmente a qualidade do serviço oferecido. O relato sobre o recebimento de orientações acerca da amamentação também se mostrou relativamente baixo, sendo maior na Região Nordeste. Conforme Toma e Rea, 28 a orientação sobre aleitamento materno deveria atingir a totalidade das gestantes, sobretudo nos municípios prioritários para a redução da mortalidade infantil, tendo em vista os benefícios da amamentação para a saúde da mulher e da criança. Ainda assim, todos os indicadores de processo para o pré-natal do PHPN ora apresentados, mostram valores médios superiores àqueles identificados para o total do país conforme recente revisão sistemática. 18

Outro aspecto preocupante foi que menos da metade das gestantes receberam indicação sobre local do parto, concordante com os resultados da pesquisa Nascer no Brasil, em que a média nacional foi de $58,7 \%$, e $49,4 \%$ na Região Norte. 5 Com relação ao parto as variáveis que mais impactaram na sua inadequação foram a não realização do parto no serviço indicado no pré-natal, a espera de mais de uma hora para internação e ausência de acompanhante em todos os momentos do parto. Quanto aos indicadores relacionados ao acesso durante o parto, verifica-se que a minoria das mulheres entrevistadas teve, seu parto no local indicado durante o pré-natal. Esse dado mostra uma desconexão entre a referência do sistema de saúde e a vida real das usuárias, uma vez que existe a Lei $\mathrm{n}^{\circ} 11.634$, desde 27 de dezembro de 2007, que garante à gestante o direito de vinculação à maternidade onde receberá assistência no âmbito do SUS. 12 Apesar da insuficiência do sistema de referência, observa-se redução da peregrinação por várias instituições para realização do parto. Pesquisa realizada no município do Rio de Janeiro em 1999-2001 revelou que mais de 30,0\% das gestantes usuárias do SUS necessitaram peregrinar por mais de uma maternidade para conseguir admissão para o parto. ${ }^{27} \mathrm{Na}$ Pesquisa Nascer no Brasil Viellas et al. 5 encontraram que $17,3 \%$ das gestantes precisaram procurar mais de uma unidade de saúde para realização do parto, com grandes variações entre as Regiões, expressando problemas na distribuição e oferta de leitos e a heterogeneidade da rede de atenção obstétrica. A demora em ser atendida no serviço foi uma condição muito relatada pelas mulheres entrevistadas, podendo estar associadas tanto à dificuldade no sistema de transporte e referência em regiões específicas, quanto à disponibilidade e à qualidade da atenção nos serviços procurados. Estes fatos caracterizam basicamente a segunda e terceira demoras de Maine relacionadas à qualidade dos serviços obstétricos. Ou seja, o retardo da mulher para chegar à instituição de saúde por problemas estruturais de transporte e de referência (segunda demora) e os problemas na disponibilidade e qualidade da atenção recebida (ou não) nos serviços de saúde (terceira demora). 30

A presença de acompanhante em todos os momentos do parto está assegurada por Lei no país desde 2005,11 mas isso ainda não está efetivamente implementado em todas as maternidades. Embora se note algum avanço nesta área, a garantia do direito das mulheres brasileiras, de estarem acompanhadas por alguém de sua livre escolha durante toda a sua estadia na maternidade, está muito longe de ser cumprido. A pesquisa Nascer no Brasil identificou $26,0 \%$ de mulheres sem acompanhante durante o parto e menos de $15,0 \%$ acompanhadas durante todo o período da internação nas regiões desse estudo, mas o dado inclui os setores conveniados com o SUS e privados, o que pode explicar, em parte, as diferenças nos valores entrados. Verificou-se também que a presença do acompanhante se correlacionava com a existência de regras institucionais claras nos hospitais sobre esse direito.

Os benefícios de uma assistência de qualidade ao parto, que inclui os direitos reprodutivos da mulher de ter uma vaga assegurada em maternidade de fácil acesso, atendimento digno (sem espera longa) e acompanhante de sua livre escolha estiveram associados à mais alta escolaridade materna. Isso indica que o sistema de saúde brasileiro não tem conseguido funcionar como um instrumento na atenuação das iniquidades sociais, ao contrário, as reproduz tanto na atenção ao pré-natal como ao parto.

No Brasil a alta cobertura do atendimento hospitalar na assistência ao parto ainda convive com obstáculos no acesso à maternidade. Persiste uma desorganização do sistema de saúde na oferta de 
leitos obstétricos e neonatais, além da precariedade na infraestrutura hospitalar e baixa qualidade técnica no atendimento obstétrico e perinatal. Observa-se maior concentração da oferta nas capitais dos Estados, onde se concentram também as instituições com maiores recursos tecnológicos.

Estudo sobre a estrutura das maternidades brasileiras revelou que a situação é particularmente preocupante em relação à atenção de emergência ao recém-nascido nas Regiões Norte e Nordeste, fato que pode se refletir nos indicadores de mortalidade neonatal. ${ }^{29}$ Nessa mesma pesquisa a mortalidade neonatal mostrou-se associada tanto às baixas condições sociais das mães quanto à inadequação da

\section{Referências}

1. Younes L, Houweling TAJ, Azad K, Costello A, Fottrell E. Estimating coverage of a women's group intervention among a population of pregnant women in rural Bangladesh. BMC Pregnancy Childbirth. 2012; 12: 60

2. Villar J, Carroli G, Khan-Neelofur D, Piaggio G, Gülmezoglu M. Patterns of Routine Antenatal care for lowrisk pregnancy (Cochrane Review). In: The Cochrane Library, Issue 4, 2008. Oxford: Update Software.

3. Barros FC, Bhutta ZA, Batra M, Hansen TN, Victora CG, Rubens CE, and the GAPPS review group. Global report on preterm and stillbirth ( 3 of 7): evidence for effectiveness of interventions. BMC Pregnancy Childbirth. 2010; 10 (Suppl. 1): S3.

4. Brasil. Ministério da Saúde. Programa de Humanização do Pré-Natal e Nascimento. Brasília, DF: Secretaria de Políticas de Saúde; 2000.

5. Viellas EF, Domingues RMSM, Dias MAB, Gama SGN, Theme-Filha MM, Costa JV, Bastos MH, Leal MC. Assistência pré-natal no Brasil. Cad Saúde Pública. 2014; 30 (Supl.): S85-S100.

6. Coutinho T, Teixeira MTB, Dain S, Sayd JD, Coutinho LM. Adequação do processo de assistência pré-natal entre as usuárias do Sistema Único de Saúde em Juiz de Fora, MG. Rev Bras Gineco Obstet. 2003; 25 (10): 717-24

7. Leal MC, Gama SGN, Ratto KMN, Cunha CB. Uso do índice de Kotelchuck modificado na avaliação da assistência pré-natal e sua relação com as características maternas e o peso do recém-nascido no Município do Rio de Janeiro. Cad Saúde Pública. 2004; 20 (Supl. 1): S63-72.

8. Coimbra LC, Silva AAM, Mochel EG, Alves MTSSB, Ribeiro VS, Aragão VMF, Bettiol H. Fatores associados à inadequação do uso da assistência pré-natal. Rev Saúde Pública. 2003; 37 (4): 456-62.

9. Brasil. Ministério da Saúde. Saúde Brasil 2011: uma análise da situação de saúde e a vigilância da saúde da mulher. Brasília, DF; 2012

10. Brasil. Lei no 11.108 de 7 de abril de 2005. Garante às parturientes o direito à presença de acompanhante durante o trabalho de parto, parto e pós-parto imediato, no âmbito do Sistema Único de Saúde - SUS. [acesso em 18 out atenção pré-natal e ao parto, à peregrinação das gestantes e inadequação das maternidades para atendimento a recém-nascidos de risco obstétrico, 32 apontando para a necessidade de intervenções coordenadas para a melhoria dos indicadores obstétricos e perinatais do país.

Este estudo, realizado em 2010, descreveu a adequação da atenção ao pré-natal e ao parto entre usuárias do SUS na Amazônia Legal e no Nordeste, a partir de indicadores estratégicos para a redução da mortalidade perinatal. Assim sendo, oportunizou a construção de uma linha de base para Rede Cegonha nos 252 municípios prioritários para a redução da mortalidade infantil no Brasil.
2014]. Disponível em: http://www.planalto.gov.br/ ccivil_03/_Ato2004-2006/2005/Lei/L11108.htm.

11. Hodnett ED, Gates S, Hofmeyr GJ, Sakala C. Continuous support for women during childbirth. Cochrane Database Syst Rev. 2011; (2): CD003766.

12. Brasil. Lei no 11.634, de 27 de dezembro de 2007. Dispõe sobre o direito da gestante ao conhecimento e a vinculação à maternidade onde receberá assistência no âmbito do Sistema Único de Saúde. [acesso em 18 out 2014]. Disponível em: http://www.planalto.gov.br/ccivil_03/ Ato2007-2010/2007/Lei/L11634.htm

13. World Health Organization, Maternal and Newborn Health/Safe Motherhood Unit. Care in normal birth: a practical guide. Genebra: World Health Organization; 1996.

14. Espanha. Ministerio de Sanidad y política Social de España. Guía de Práctica Clínica sobre la Atención al Parto Normal. Madri: Ministerio de Sanidad y Política Social de España; 2010.

15. Brasil. Ministério da Saúde. Portaria ${ }^{\circ} 1.459$ de 24 de julho de 2011. Institui, no âmbito do Sistema Único de Saúde - SUS - a Rede Cegonha. [acesso em 18 out 2014]. Disponível em: http://bvsms.saude.gov.br/bvs/saudelegis/ gm/2011/prt1459_24_06_2011.html.

16. Leal MC, Pereira APE Domingues RMSM, Theme-Filha MM, Dias MAB, Nakamura-Pereira M, Bastos MH, Gama SGN. Intervenções obstétricas durante o trabalho de parto e parto em mulheres brasileiras de risco habitual. Cad Saúde Pública. 2014; 30 (Supl.): S17-32.

17. Brasil. Ministério da Saúde. Secretaria de Ciência, Tecnologia e Insumos Estratégicos. Departamento de Ciência e Tecnologia. Avaliação da atenção ao pré-natal, ao parto e aos menores de um ano na Amazônia Legal e no Nordeste, Brasil, 2010. Brasília, DF. 2013. Cap. 2. Métodos, pp. 190-32. [acesso em 4 mai 2014]. Disponível em: http://bvsms.saude.gov.br/bvs/publicacoes/livro avaliacao_da_atencao_ao_pre-natal_web.pdf

18. Andreucci CB, Cecatti JG. Desempenho de indicadores de processo do Programa de Humanização do Pré-Natal e Nascimento no Brasil: uma revisão sistemática. Cad Saúde Pública. 2011; 27 (6): 1053-64. 
19. Brasil. Ministério da Saúde. Pesquisa Nacional de Demografia e Saúde da Criança e da Mulher - PNDS 2006 dimensões do processo reprodutivo e da saúde da criança. Brasília, DF; 2009.

20. Victora CG, Aquino EM, do Carmo Leal M, Monteiro CA, Barros FC, Szwarcwald CL. Maternal and child health in Brazil: progress and challenges. Lancet. 2011; 377 (9780) 1863-76.

21. Wehby GL, Murray JC, Castilla EE, Lopez-Camelo JS, Ohsfeldt RL. Prenatal care effect ivenesse and utilization in Brazil. Health Policy Plan. 2009; 24: 175-88.

22. Debiec KE, Paul KJ, Mitchell CM, Hitti JE. Inadequate prenatal care and risk of preterm delivery among adolescents: a retrospective study over 10 years. Am J Obstet Gynecol. 2010; 203: 122. e1-6.

23. Serruya SJ; Lago TG; Cecatti JG. Avaliação preliminar do programa de humanização no pré-natal e nascimento no Brasil. Rev Bras Ginecol Obstet. 2004; 26 (7): 517-25

24. Coutinho T, Monteiro MFG, Sayd JD, Teixeira MTB, Coutinho CM, Coutinho LM. Monitoramento do processo de assistência pré-natal entre as usuárias do Sistema Único de Saúde em município do Sudeste brasileiro. Rev Bras Ginecol Obstet. 2010; 32: 563-9.

25. Domingues RMSM, Hartz ZMA, Dias MAB, Leal MC. Avaliação da adequação da assistência pré-natal na rede SUS do Município do Rio de Janeiro, Brasil. Cad Saúde Pública. 2012; 28 (3): 425-37.

26. Araújo CL, Shimizu HE, Sousa AI, Hamann EM. Incidence of congenital syphilis in Brazil and its relationship with the Family Health Strategy. Rev Saúde Pública. 2012; 46 (3): 479-86.

Recebido em 29 de abril de 2014

Versão final apresentada em 31 de outubro de 2014

Aprovado em 26 de dezembro de 2014
27. Schoeps D, Almeida MF, Alencar GP, França Jr I, Novaes HMD, Siqueira AF, Campbell O, Rodrigues LC. Fatores de risco para mortalidade neonatal precoce. Rev Saúde Pública. 2007; 41 (6): 1013-22.

28. Toma TS, Rea, MF. Benefícios da amamentação para a saúde da mulher e da criança: um ensaio sobre as evidências. Cad Saúde Pública. 2008; 24 (Supl. 2): S235-46.

29. Leal MC, Gama SGN, Campos MR, Cavalini LT, Garbayo LS, Brasil CLP, Szwarcwald CL. Fatores associados à morbi-mortalidade perinatal em uma amostra de maternidades públicas e privadas do Município do Rio de Janeiro, 1999-2001. Cad Saúde Pública. 2004; 20 (Supl. 1): S20-33.

30. Pacagnella RC, Cecatti JG, Osis MJ, Souza JP. The role of delays in severe maternal morbidity and mortality: expanding the conceptual framework. Reprod Health Matters. 2012; 20 (39): 155-63.

31. Bittencourt SDA, Reis LGC, Ramos MM, Rattner D, Rodrigues PL, Neves DCO, Arantes SL, Leal MC. Estrutura das maternidades: aspectos relevantes para a qualidade da atenção ao parto e nascimento. Cad Saúde Pública. 2014; 30 (Supl.): S208-19.

32. Lansky S, Friche AAL, Silva AAM, Bittencourt SDA, Carvalho ML, Frias PG, Cavalcante RS, Cunha AJAC. Pesquisa Nascer no Brasil: perfil da mortalidade neonatal e avaliação da assistência à gestante e ao recém-nascido. Cad Saúde Pública. 2014; 30 (Supl.): S192-207. 